\title{
Auxiliary diagnostic value of p16 amplification combined with the detection of heterozygous and homozygous loss for urothelial carcinoma
}

\author{
XIAOPENG MAO ${ }^{1,2^{*}}$, BAIMOU LI ${ }^{1,2^{*}}$, YING LIANG $^{3}$, SHUHUA LI $^{1}$, \\ JIANWEN ZHOU ${ }^{1}$, QIONG HE ${ }^{1}$, NENG JIANG ${ }^{1}$, YANGSHAN CHEN ${ }^{1}$, YU SUN ${ }^{1}$, \\ YONGMEI CUI $^{1}$, WENTING JIANG ${ }^{1}$, HAN WANG $^{1}$, LIANTANG WANG ${ }^{1}$ and ZUNFU KE ${ }^{1}$
}

Departments of ${ }^{1}$ Pathology and ${ }^{2}$ Urology Surgery, The First Affiliated Hospital, Sun Yat-sen University, Guangzhou, Guangdong 510080; ${ }^{3}$ Department of Nephrology, General Hospital of Guangzhou Military Command of PLA, Guangzhou, Guangdong 510010, P.R. China

Received August 11, 2017; Accepted January 10, 2018

DOI: $10.3892 / \mathrm{ol} .2018 .8137$

\begin{abstract}
The present study aimed to investigate the significance of detecting cyclin dependent kinase inhibitor 2A (p16) gene aberrations in the diagnosis of urothelial carcinoma (UC) using fluorescence in situ hybridization (FISH). A total of 77 voided urine specimens from 65 patients with UC and 12 patients with benign urinary disease were recruited into the current study. Under a fluorescence microscope, cells with large and irregular nuclei were assessed for chromosomal aberrations. The positive rate of p16 amplification in UC samples was $32.3 \%$ (21/65), which was significantly higher than that in benign urinary disease samples $(16.7 \%, 2 / 12 ; \mathrm{P}<0.05)$. Heterozygous and homozygous loss of p16 was identified in $12(18.5 \%)$ and $23(35.4 \%)$ patients with UC, respectively; p16 expression in the remainder of patients was normal. In addition, as tumor stage or grade advanced, the positive rate of 16 aberrations also increased significantly $(\mathrm{P}<0.05)$. In conclusion, p16 gene aberrations may serve important roles in the auxiliary diagnosis of UC by FISH and could be utilized to monitor UC progression.
\end{abstract}

\section{Introduction}

In the Unites States and China, the incidence and mortality rates of urothelial carcinoma (UC) increases yearly $(1,2)$. UC is a malignant tumor originating from urinary tract epithelium,

Correspondence to: Dr Zunfu Ke, Department of Pathology, The First Affiliated Hospital, Sun Yat-sen University, 58 Zhongshan Second Road, Guangzhou, Guangdong 510080, P.R. China

E-mail: kezunfu@mail.sysu.edu.cn

${ }^{*}$ Contributed equally

Key words: urothelial carcinoma, fluorescence in situ hybridization, p16, deletion, amplification and primarily consists of renal pelvis, ureter and bladder cancer $(3,4)$. The majority of cases of UC arise in the bladder, although renal pelvis and ureter cancer account for $\sim 10 \%$ of cases of UC (3). The origins and pathogenesis of these diseases is similar; however, they possess distinct symptoms, including microscopic and macroscopic painless hematuria and lower urinary tract symptoms (4). UC has a high rate of invasion, recurrence and metastasis (5), making it important to detect $\mathrm{UC}$ from voided urine specimens as early as possible.

At present, radiographic testing, urine cytology, cystoscopy and ureteroscopy are the most common methods for the diagnosis and follow-up of patients with UC $(6,7)$. A biopsy performed using cystoscopy or ureteroscopy is the gold standard for UC diagnosis; however, the procedure is invasive and causes patient discomfort (8). Cytology is a non-invasive method with a high specificity; however, its sensitivity is low, making the early detection of cancer by this method inefficient (9). Although ultrasound or computed tomography can identify the mass and consequential dilatation of the urinary system, the sensitivity of these techniques depends on the quality of equipment and experience of the clinician (6). Endoscopy is an invasive method from which complications may arise, including hemorrhage and infection (8). Thus, the use of cytology and these imaging techniques are limited to the detection of small tumors and low-stage lesions. Therefore, it is necessary to develop a non-invasive and highly sensitive method for the detection of UC in its early stages.

Tumor-associated chromosomal aberrations and gene alterations are the focus of present research into tumor pathogenesis and progression (10-12). Previous studies have indicated that the cause of the majority of urothelial carcinoma cases is associated with chromosomal aberration $(11,13)$. Aneuploidy and chromosomal aberration (deletion or amplification), particularly on chromosomes 3, 7, 17 and 9 (the location of cyclin dependent kinase inhibitor 2A (CDKN2A, also known as p16), are positively associated with tumor grade (14). Fluorescence in situ hybridization (FISH) can be used to detect the aforementioned cytogenetic changes in voided urine samples to achieve the early diagnosis of UC, which is a method approved 
by the US Food and Drug Administration for UC detection (13). At present, this method is widely used in Europe and the United States for the clinical detection of UC; however, the focus of the current study is primarily on abnormalities to chromosomes 3, 7 and 17 (deletion, haploidy or polyploidy) and the deletion of chromosome $9(15,16)$. Additionally, the present study aimed to assess the role of p16 amplification in the development of urothelial carcinoma and the association with clinical stage and classification.

The p16 gene, also known as multiple tumor suppressor 1 and cyclin-dependent kinase-4 (CDK4) inhibitor, is a multi-tumor suppressor gene associated with certain types of human malignant tumor $(10,17)$. p16 is located on the short arm of human chromosomal 9 (9p21), is $8.5 \mathrm{~kb}$ in length and is comprises 3 exons and 2 introns. The protein encoded by p16 has a molecular weight of $16 \mathrm{kDa}$ and is composed of 148 amino acids. As an inhibitor of CDK4, p16 is a negative regulator of cell proliferation and a tumor suppressor gene that is directly involved in cell cycle progression (17). The previous diagnostic criteria only assessed the heterozygous and homozygous deletion of p16 without considering p16 amplification $(13,16)$; however, it was identified that that p16 amplification is a frequent event in ongoing surceillance by our research group, making it necessary to investigate the diagnostic value of detecting p16 amplification in combination with p16 heterozygous and homozygous deletion. The present study aimed to detect p16 aberrations combining the aforementioned targets (p16 amplification, heterozygous and homozygous deletion) by FISH assay and to evaluate the clinical significance of genetic alteration to p16, particularly polyploidy, in the diagnosis of UC.

\section{Materials and methods}

Patients and samples. The study was conducted at the Department of Urology and Pathology, The First Affiliated Hospital, Sun Yat-sen University (Guangzhou, China) between November 2009 and March 2017. Voided urine specimens from 77 patients (45 males and 32 females; mean age, 64.2 years; age range, 28-86 years) were collected in the morning for analysis using FISH assays. The patients recruited were those with urinary symptoms such as hematuria and lower urinary tract symptoms, but the most common methods mentioned above (radiographic testing, urine cytology, cystoscopy and ureteroscopy) did not distinguish between benign or malignant cells. Those who were diagnosed or treated in the past were excluded. The cohort consisted of 65 samples from patients with urothelial carcinoma (40 males and 25 females; mean age, 64.8 years; age range, 28-86 years) and 12 samples from patients with urinary benign disease as control group (5 males and 7 females; mean age, 61.1 years; age range, 34-74 years; 3 with prostatic hyperplasia, 3 with cyst and 6 with nephrotic syndrome or nephritis). Tumors were localized to the renal pelvis (37 cases), ureter (15 cases) and bladder (13 cases).

All patients were diagnosed whether they were UC by using pathology as the gold standard using specimens obtained via surgical resection. In hematoxylin and eosin (H\&E) staining, the tissues were first fixed in $10 \%$ formalin for $30 \mathrm{~min}$ at room temperature. Then, paraffin-embedded slides were obtained after a series of steps including dehydration $(50 \%, 70 \%, 80 \%$, $95 \%, 100 \%, 100 \%$ alcohol, each concentration for $30 \mathrm{~min}$ ), paraffin embedding and sectioning. The slides were stained in hematoxylin for $5 \mathrm{~min}$ and eosin for $1 \mathrm{~min}$ at room temperature.

The present study was approved by the Medical Ethics Committee of Sun Yat-sen University (Guangzhou, Guangdong, China). Written informed consent was obtained from all patients prior to enrollment.

FISH assay. Urine samples were collected in the morning (at least $200 \mathrm{ml}$ ) for FISH analysis. The voided urine samples were centrifuged at $2,582 \mathrm{x}$ g for $5 \mathrm{~min}$ at room temperature and incubated in a hypotonic solution $(0.075 \mathrm{M} \mathrm{KCl})$ at room temperature for $20 \mathrm{~min}$. The cells were fixed with mixed solution (methanol: Acetic acid, 2:1) twice at room temperature, each for $10 \mathrm{~min}$. A total of 4 slides were produced from the re-suspended cells of each patient and were stored until use for subsequent FISH assays.

The specific probe kit (9p21, p16 probe; cat. no. F01008-02) used in the present study was purchased from Beijing GP Medical Technologies, Ltd., Beijing, China. White blood cells (WBCs) from healthy participants were utilized as controls. A probe targeting centromere and p16 was used to ensure that the experimental method and procedure were correct. Absence of signal was likely to due to deletion of p16 rather than a hybridization technical failure. According to the kit protocol, slides were washed with $2 \mathrm{X}$ saline sodium citrate (SSC) and then dehydrated in a series of ethanol washes ( 70,85 and $100 \%$ for 2 min each at room temperature). A $10-\mu 1$ probe mixture was then added to each slide and samples were denatured at $75^{\circ} \mathrm{C}$ for $5 \mathrm{~min}$, followed by $42^{\circ} \mathrm{C}$ overnight in a Denaturation \& Hybridization System (StatSpin Thermobrite, USA). Next, the slides were treated with $2 \mathrm{X}$ SSC for $20 \mathrm{~min}$ and $2 \mathrm{X} \mathrm{SSC} / 0.1 \% \mathrm{NP}-40$ for $5 \mathrm{~min}$ at $42^{\circ} \mathrm{C}$. DAPI was used for counterstaining. The fluorescent hybridization signal was observed under a fluorescence microscope (low magnification, $\mathrm{x} 100$; high magnification, $\mathrm{x} 1,000)$ and analyzed by a software (VideoTesT-FISH 2.0, VideoTesT).

A uniform field of view was utilized to analyze atypical epithelial cells with large and irregular nuclei at a low magnification (x100). Cells that overlapped, were incomplete, had large quantities of cytoplasm or produced weak hybridization signals were excluded. Close intracellular signals, whose distance was $<2$ hybridization points apart, were considered one signal. Those cells with 2 signals were judged as normal cells without p16 deletion or amplification. In addition, cells that produced $\geq 3$ signals were deemed to demonstrate triploidy or polyploidy. Besides, cells that produced only 1 or 0 signals were considered to exhibit heterozygous or homozygous deletions, respectively. If the number of aberrant cells exceeded $10 \%$ of the total cell number, the specimen was considered to have a positive FISH result. A total of 100 nuclei were studied per slide. The results of amplification, heterozygous and homozygous deletion were combined together to diagnose UC. If any aberration occurred in these samples, the FISH result was considered positive. The absence of aberration was indicative of a negative FISH result.

Statistical analysis. The significance of intergroup differences were calculated using the $\chi^{2}$ test. The rate of p16 
Table I. FISH results (p16 amplification) for patients with histologically diagnosed urothelial carcinoma.

\begin{tabular}{|c|c|c|c|c|c|c|c|}
\hline No. & Sex & Age & Tumor stage & Tumor grade & Location & FISH results & Type of aberration \\
\hline 1 & $\mathrm{~F}$ & 69 & pT2 & High & Renal pelvis & Positive & Amplification \\
\hline 2 & M & 75 & pT2 & High & Ureter & Positive & Homozygous \\
\hline 3 & M & 46 & pT2 & High & Renal pelvis & Positive & Amplification \\
\hline 4 & M & 67 & pT2 & High & Renal pelvis & Positive & Heterozygous \\
\hline 5 & M & 79 & pT1 & High & Bladder & Positive & Homozygous \\
\hline 6 & M & 49 & pT3 & High & Renal pelvis & Positive & Homozygous \\
\hline 7 & M & 71 & pT1 & Low & Bladder & Negative & - \\
\hline 8 & M & 45 & pT2 & High & Renal pelvis & Positive & Amplification \\
\hline 9 & $\mathrm{~F}$ & 74 & pT1 & Low & Renal pelvis & Positive & Homozygous \\
\hline 10 & M & 68 & pT1 & Low & Bladder & Positive & Homozygous \\
\hline 11 & $\mathrm{~F}$ & 59 & pT2 & Low & Renal pelvis & Positive & Homozygous \\
\hline 12 & $\mathrm{M}$ & 31 & pT1 & Low & Bladder & Negative & - \\
\hline 13 & M & 61 & pT3 & High & Renal pelvis & Positive & Homozygous \\
\hline 14 & $\mathrm{~F}$ & 67 & pT1 & Low & Renal pelvis & Negative & - \\
\hline 15 & M & 78 & pT3 & High & Ureter & Positive & Homozygous \\
\hline 16 & M & 58 & pT4 & High & Ureter & Positive & Homozygous \\
\hline 17 & $\mathrm{M}$ & 76 & pT1 & High & Ureter & Positive & Homozygous \\
\hline 18 & $\mathrm{~F}$ & 73 & pT1 & Low & Bladder & Negative & - \\
\hline 19 & M & 77 & pT1 & High & Renal pelvis & Positive & Homozygous \\
\hline 20 & $\mathrm{M}$ & 66 & pT2 & High & Renal pelvis & Positive & Homozygous \\
\hline 21 & $\mathrm{~F}$ & 72 & pT1 & Low & Renal pelvis & Positive & Amplification \\
\hline 22 & $\mathrm{~F}$ & 56 & pT3 & High & Ureter & Positive & Amplification \\
\hline 23 & M & 74 & pT1 & Low & Ureter & Negative & - \\
\hline 24 & M & 72 & pT1 & Low & Bladder & Negative & - \\
\hline 25 & M & 74 & pT2 & Low & Renal pelvis & Positive & Heterozygous \\
\hline 26 & $\mathrm{M}$ & 43 & pT2 & High & Renal pelvis & Positive & Homozygous \\
\hline 27 & $\mathrm{~F}$ & 86 & pT2 & High & Renal pelvis & Positive & Amplification \\
\hline 28 & $\mathrm{M}$ & 67 & pT2 & High & Ureter & Positive & Amplification \\
\hline 29 & M & 59 & pT1 & High & Renal pelvis & Positive & Homozygous \\
\hline 30 & M & 76 & pT1 & High & Bladder & Positive & Amplification \\
\hline 31 & M & 71 & pT2 & High & Ureter & Positive & Homozygous \\
\hline 32 & M & 67 & pT1 & Low & Bladder & Negative & - \\
\hline 33 & $\mathrm{~F}$ & 74 & pT1 & High & Ureter & Positive & Heterozygous \\
\hline 34 & $\mathrm{~F}$ & 68 & pT2 & High & Renal pelvis & Positive & Amplification \\
\hline 35 & M & 60 & pT2 & High & Renal pelvis & Positive & Amplification \\
\hline 36 & M & 53 & pT1 & Low & Bladder & Positive & Amplification \\
\hline 37 & $\mathrm{~F}$ & 70 & pT2 & High & Ureter & Negative & - \\
\hline 38 & $\mathrm{~F}$ & 67 & pT2 & High & Renal pelvis & Positive & Amplification \\
\hline 39 & M & 60 & pT2 & High & Renal pelvis and ureter & Positive & Heterozygous \\
\hline 40 & M & 77 & pT2 & High & Bladder & Positive & Amplification \\
\hline 41 & $\mathrm{~F}$ & 59 & pT3 & High & Ureter & Positive & Heterozygous \\
\hline 42 & M & 55 & pT1 & Low & Bladder & Positive & Amplification \\
\hline 43 & $\mathrm{~F}$ & 49 & pT2 & High & Renal pelvis & Positive & Amplification \\
\hline 44 & $\mathrm{~F}$ & 74 & pT2 & High & Ureter & Positive & Homozygous \\
\hline 45 & $\mathrm{~F}$ & 73 & pT3 & High & Renal pelvis & Positive & Heterozygous \\
\hline 46 & $\mathrm{~F}$ & 62 & pT2 & High & Renal pelvis & Positive & Amplification \\
\hline 47 & M & 50 & pT2 & High & Bladder & Positive & Heterozygous \\
\hline 48 & $\mathrm{~F}$ & 53 & pT2 & High & Renal pelvis & Positive & Homozygous \\
\hline 49 & $\mathrm{~F}$ & 82 & pT1 & High & Renal pelvis & Positive & Homozygous \\
\hline 50 & M & 59 & pT1 & Low & Renal pelvis & Positive & Amplification \\
\hline 51 & $\mathrm{~F}$ & 58 & pT1 & Low & Ureter and bladder & Negative & - \\
\hline 52 & M & 77 & pT4 & High & Renal pelvis & Positive & Amplification \\
\hline
\end{tabular}


Table I. Continued.

\begin{tabular}{lcccclcc}
\hline No. & Sex & Age & Tumor stage & Tumor grade & \multicolumn{1}{c}{ Location } & FISH results & Type of aberration \\
\hline 53 & F & 60 & pT2 & High & Renal pelvis & Positive & Homozygous \\
54 & M & 79 & pT2 & High & Renal pelvis and ureter & Positive & Amplification \\
55 & F & 62 & pT2 & High & Renal pelvis & Positive & Amplification \\
56 & F & 71 & pT2 & High & Renal pelvis & Positive & Homozygous \\
57 & M & 64 & pT2 & High & Renal pelvis & Positive & Homozygous \\
58 & M & 85 & pT2 & High & Bladder & Positive & Heterozygous \\
59 & M & 51 & pT1 & Low & Renal pelvis & Positive & Amplification \\
60 & M & 71 & pT3 & High & Ureter & Positive & Heterozygous \\
61 & F & 68 & pT2 & High & Renal pelvis & Positive & Heterozygous \\
62 & M & 28 & pT1 & Low & Renal pelvis & Positive & Homozygous \\
63 & F & 73 & pT2 & High & Ureter & Positive & Homozygous \\
64 & M & 66 & pT2 & High & Renal pelvis & Positive & Heterozygous \\
65 & M & 49 & pT3 & High & Renal pelvis & Positive & Heterozygous \\
\hline
\end{tabular}

F, female; M, male; FISH, fluorescence in situ hybridization.

aberrations between UC and control groups was analyzed and the association between FISH result and tumor stage, grade (Tumor-Node-Metastasis classification by World Health Organization/International Society of Urology Pathologists, 2004) (18) and location was assessed. To evaluate the auxiliary diagnostic value of p16 in $\mathrm{UC}$, a receiver operating characteristic (ROC) curve was performed to compare the diagnostic power of p16 deletion, p16 amplification alone and the two in combination. The ROC area under the curve (AUC) reflects the diagnostic value of different methods. The larger the AUC is, the more valuable the test method shows. The ROC curves were created by 3 groups of data (p16 deletion, p16 amplification and the combination of the two). Statistical analysis was performed using SPSS software, version 20.0 (IBM Corp., Armonk, NY, USA). For all statistical tests, $\mathrm{P}<0.05$ was considered to indicate a statistically significant difference.

\section{Results}

Characteristics of p16 aberrations in patients with UC. In the present study, 65 patients with UC and 12 patients with benign disease were analyzed. The results of FISH clinical data analysis for all patients with UC are presented in in Table I. According to the pathological Tumor-Node-Metastasis classification (19) of renal pelvis, ureter and bladder carcinoma, the 65 resected tumors were classified as non-muscle-invasive (pTa, pT1) in 23 cases (35.4\%) and muscle-invasive (pT2-4) in 42 cases $(64.6 \%)$. The tumor grade was low in 18 cases (27.7\%) and high in 47 cases (72.3\%). Epithelioid cells with 2 red signals in their nuclei were confirmed as normal. In addition, p16 heterozygous deletions, homozygous deletions and amplification polyploidy was detected with 1,0 and $>2$ red signals, respectively. Furthermore, the control probe targeting the centromeres in WBCs demonstrated that the experimental method and procedure were correct; the green signals indicated centromeres in WBCs (Fig. 1). p16 gene amplification was detected in 21/65 patients (32.3\%) with UC and in 2 of 12 patients (16.7\%) with benign disease, respectively (Table II). In addition, p16 heterozygous and homozygous loss was identified in 12 (18.5\%) and 23 cases (35.4\%) of UC, respectively. The expression of p16 was normal in the remaining cases. The results of amplification, heterozygous loss and homozygous deletion were combined into a single diagnostic criterion. p16 aberrations were found in 56 cases of 65 cases with urothelial carcinoma, so the sensitivity of FISH for UC is $86.2 \%$ (56/65). And in 12 patients with benign disease, 9 patients showed normal signals for FISH results, so the specificity was $75.0 \%(9 / 12)$. Therefore, the overall sensitivity and specificity of the FISH analysis using the p16 probe was 86.2 and $75.0 \%$, respectively.

There were several incidences in which the radiographic testing of patients with UC was normal, but p16 detection was aberrant. For instance, there was a unique case in which the radiographic testing indicated that the disease was benign, but FISH results demonstrated an amplification of p16. The patient was finally diagnosed with a high-grade urothelial carcinoma by the gold standard H\&E staining method (Fig. 2).

Association between p16 FISH status and clinical pathological parameters. The association between FISH results and pathological parameters, including stage, grade and tumor location, are presented in Table III. According to the aforementioned criterion, FISH positive rates between different stages, grades and locations were compared. As the level of stage or grade increased, the positive rate of p16 aberrations increased significantly $(\mathrm{P}<0.05)$. Furthermore, the tumors of 37 cases $(56.9 \%)$ were located in the renal pelvis, 15 in the ureter $(23.1 \%)$ and 13 in the bladder (20.0\%). The positive rates among these locations were also significantly different $(\mathrm{P}<0.05)$. p16 aberrations were found in 15 cases of 23 cases with pTa-pT1, so the sensitivity of FISH for pTa-pT1 is $65.2 \%$ (15/23). Similarly, we can calculate others' sensitivity with the same methods. For pTa-pT1: $41 / 42$ (97.6\%), for low-grade: 

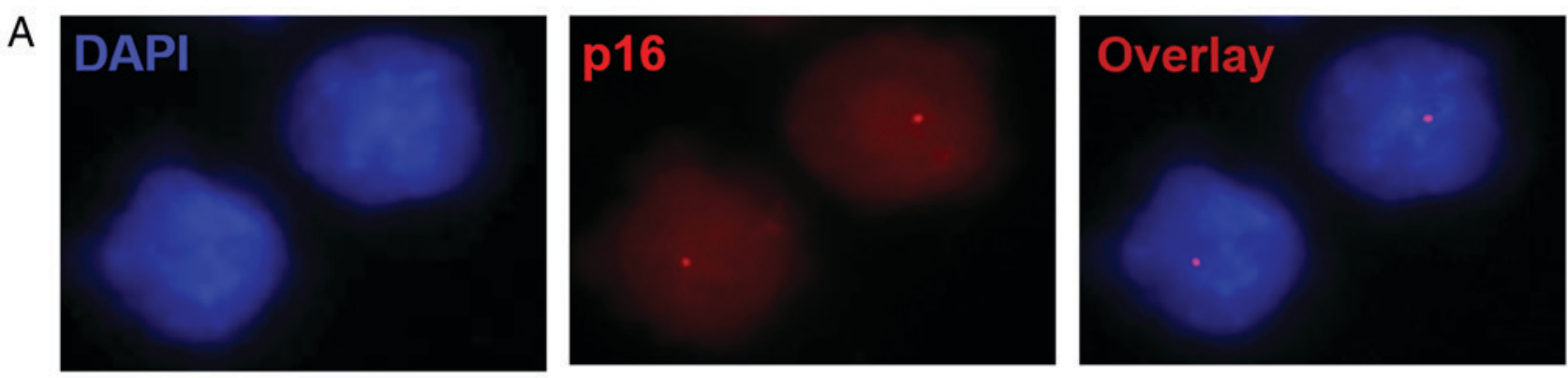

$\mathrm{B}$
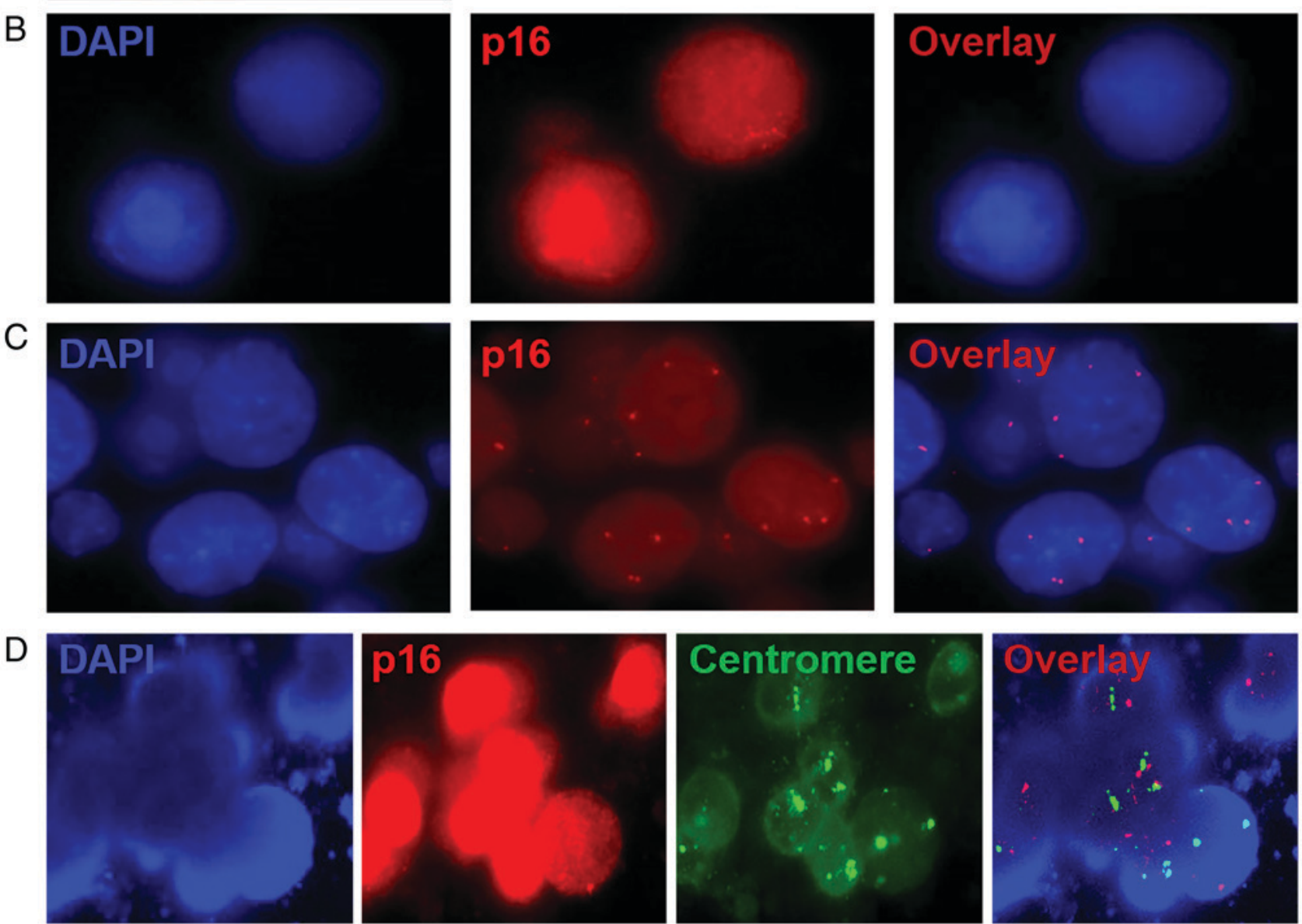

Figure 1. Various abnormal signal patterns were observed in urine samples. (A) Heterozygous deletion of p16: A single signal was observed for 9p21; (B) Homozygous deletion of p16: No signal was observed for 9p21; (C) Amplification of p16: >2 signals were detected for 9p21; (D) The fluorescence in situ hybridization results of the control group containing a p16 probe and probe targeting centromere in WBCs (p16, red; centromere, green). p16, cyclin dependent kinase inhibitor 2A (magnification, x1,000, fluorescence microscope: Olympus BX51).

10/18 (55.6\%), for high-grade: 46/47(97.9\%). In summary, the sensitivity of FISH for pTa-pT1 and pT2-pT4 tumors was 65.2 and $97.6 \%$, respectively. The sensitivity of FISH for low- and high-grade tumors was 55.6 and $97.9 \%$, respectively.

Diagnostic value of 16 aberrations. The results demonstrated that the AUC values for p16 deletion, p16 amplification and the combination were $0.728,0.578$ and 0.806 , respectively. The AUC of groups were compared by SPSS software (version 20.0) and the difference between the p16 deletion and combination of deletion and amplification groups was statistically significant $(\mathrm{P}<0.05)$. In addition, the $95 \%$ confidence intervals of their AUCs were 0.594-0.861 and 0.654-0.957, respectively. According to these data, the diagnostic value of p16 deletion and amplification when combined is higher than for any indicator alone (Fig. 3).

\section{Discussion}

$\mathrm{UC}$ is the most common malignant tumor of the urinary system. There are $\sim 100,000$ cases diagnosed and 30,000 incidences of mortality due to UC each year in United States alone (2). The high incidence and recurrence rate associated with the disease make early diagnosis and follow-up after surgery particularly important $(1,2)$. Previous studies (6-8) have demonstrated that chromosomes and their fragments, including chromosomes 3, 7 and 17 with the p16 (9p21) locus, may be associated with certain non-random changes in the development of UC. Chromosomal aberration is the primary cause of UC genesis and development; therefore, its detection in UC cells provides valuable information with regards to clinical diagnosis and therapeutic action. On the basis of their high sensitivity and specificity, FISH assays are one of 
Table II. Fluorescence in situ hybridization results for patients with urothelial carcinoma and benign disease.

\begin{tabular}{lccccccr}
\hline & \multicolumn{3}{c}{ Chromosomal aberration } & & & \\
\cline { 2 - 5 } Type & Heterozygous & Homozygous & Amplification & & Normal & Total & P-value \\
\hline Urothelial carcinoma & 12 & 23 & 21 & 9 & 65 & $<0.05$ \\
Benign urinary disease & 1 & 0 & 2 & 9 & 12 & \\
\hline
\end{tabular}

All chromosomal aberrations (heterozygous, homozygous and amplification) were combined to compare with the normal signals using $\chi^{2}$ test.
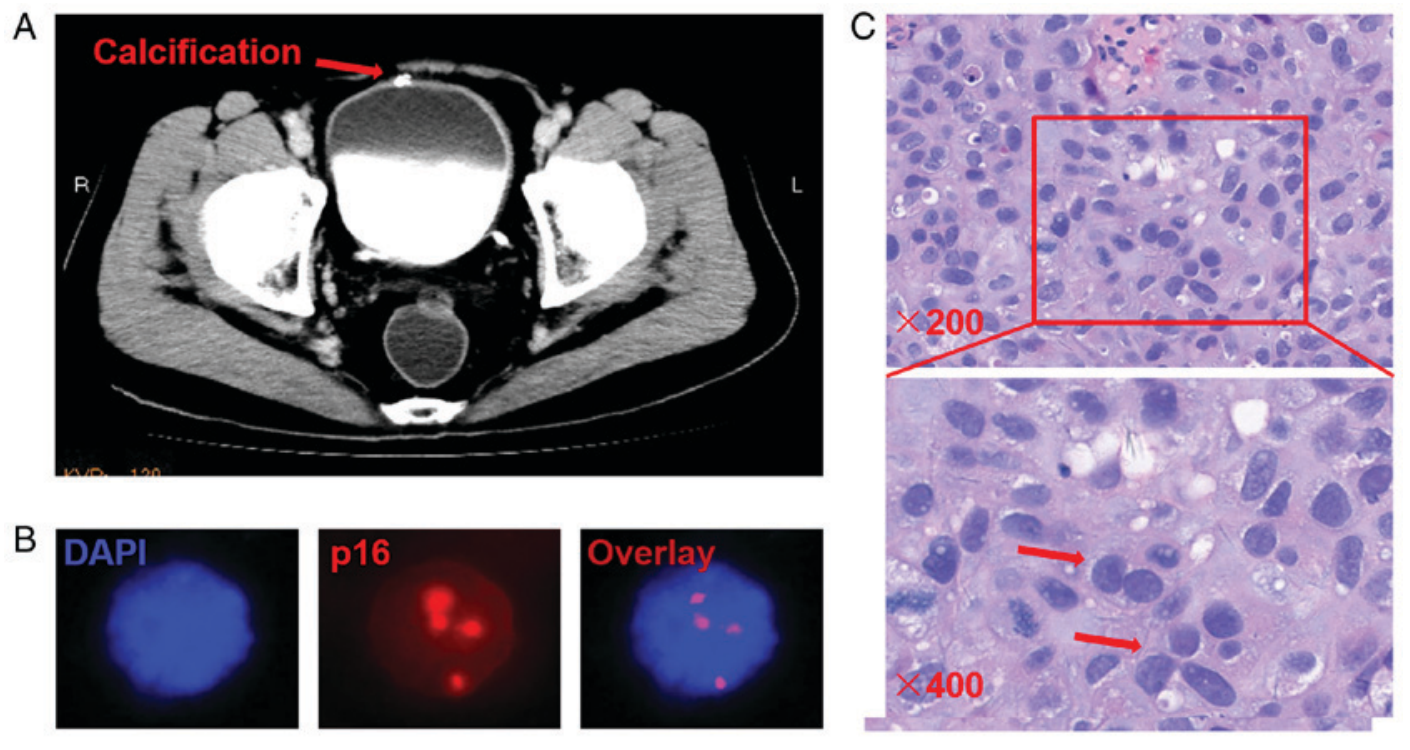

Figure 2. Abdominal computed tomography, fluorescence in situ hybridization results and H\&E staining of a specific patient. (A) Abdominal computed tomography presented bladder calcification, which indicated chronic inflammation of the bladder; (B) p16 amplification was detected in the urine sample of the patient (magnification, x1,000); (C) H\&E staining indicated that resected tissue obtained from surgery was high-grade urothelial carcinoma. Red arrows denote typical urothelial carcinoma cells. H\&E, hematoxylin and eosin; p16, cyclin dependent kinase inhibitor $2 \mathrm{~A}$.

the most common methods used in molecular genetic research, and are widely used for the study of chromosome number and structural distortion in UC. Although chromosomal aberration is identified in UC, chromosomes 3, 7, 9 and 17 are primarily used for its clinical diagnosis $(9,15)$. Chromosomal aberrations consist of deletion, haploidy and polyploidy, and the change to 9 p21 (p16) is specifically associated with deletion $(13,16)$; however, studies assessing the role of p16 gene amplification in UC are limited (20).

The present study utilized the p16 gene as a specific probe to detect the status of chromosome 9 in voided urine specimens from 65 cases of UC and 12 cases of benign disease. FISH assays were performed to evaluate the value of p16 deletion and polyploidy in the clinical diagnosis and degree of progression of UC. The positive rate of p16 deletion in UC was $53.8 \%$ (18.5\% heterozygous deletion and $35.4 \%$ homozygous deletion). Chromosome 9 incorporates a number of tumor suppressor genes, including cyclin dependent kinase inhibitor 2B, p16 and TSC complex subunit 1, and its deletion may be an early event in the tumorigenic pathway that leads to UC development $(11,21)$. In addition, chromosome 9 deletion is associated with the recurrence and progression of bladder cancer (22). The heterozygous and homozygous loss of the p16 locus in UC is detected using FISH assays and its positive rate in previous studies is $\sim 50 \%(16,23)$, which is similar to the results of the present study.

However, in the current study, p16 gene amplification accounted for some of the aberrations detected on chromosome 9 , which is not consistent with previous studies $(8,16)$. P16 polyploidy in UC was $32.3 \%$ (21/65), which was significantly higher than that in urinary benign disease $(16.7 \% ; 2 / 12$; $\mathrm{P}<0.05)$. As tumor stage and grade increased, the positive rate of FISH results also increased. When the tumor stage was pT2-pT4 or the tumor grade was high, the sensitivity of FISH assays for diagnosing UC by using a single p16 probe was $>95 \%$. The results indicated that p16 aberrations, including polyploidy, were closely associated with the classification and stage of UC. In addition, chromosomal aberrations may be an initial step in UC development, which may be valuable in the prediction of UC progression. The results of the current study are also similar to those of a previous study, which demonstrated the importance of alterations to chromosomes 3, 7, 17 and p16 in UC (12). In addition, Berggren de Verdier et al (20) demonstrated that UC cases with multiple duplications at chromosome 9p21 were associated with poor survival.

p16 amplification was detected in $32.3 \%$ patients with $\mathrm{UC}$ in the present study. This result may be explained by two theories. Firstly, although p16 is a tumor suppressor 
Table III. Fluorescence in situ hybridization results of urothelial carcinoma in various stages and grades.

\begin{tabular}{lcccc}
\hline $\begin{array}{l}\text { Pathological } \\
\text { parameters }\end{array}$ & Positive & Negative & Total & P-value \\
\hline $\begin{array}{l}\text { Stage } \\
\text { pTa-pT1 }\end{array}$ & 15 & 8 & 23 & $<0.05$ \\
pT2-pT4 & 41 & 1 & 42 & \\
Grade & & & & $<0.05$ \\
Low & 11 & 8 & 18 & \\
High & 46 & 1 & 47 & \\
Location & & & & $<0.05$ \\
Renal pelvis & 36 & 1 & 37 & \\
Ureter & 12 & 3 & 15 & \\
Bladder & 8 & 5 & 13 & \\
\hline
\end{tabular}

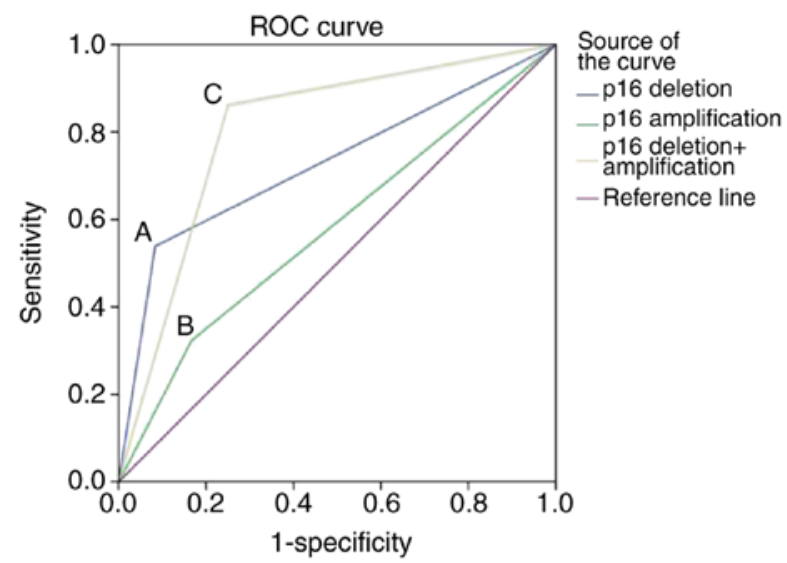

Diagonal segments are produced by ties

Figure 3. ROC curve of p16 deletion, p16 amplification and the two combined for the diagnosis of urothelial carcinoma. A, The sensitivity and specificity of p16 deletion were 53.8 and $91.6 \%$, respectively, with an AUC of 0.728 (95\% confidence interval, 0.594-0.861). B, The sensitivity and specificity of p16 amplification were 32.3 and $83.3 \%$, respectively, with an AUC value of 0.578 (95\% confidence interval, $0.412-0.745$ ). C, p16 deletion in combination with amplification produced a sensitivity and specificity of 86.2 and $75.0 \%$, respectively, with an AUC of 0.806 (95\% confidence interval, 0.654-0.957). ROC, receiver operating characteristic; AUC, area under the curve; p16, cyclin dependent kinase inhibitor $2 \mathrm{~A}$.

gene, its amplification may only be an intermediate step in the progression of UC and an unknown regulatory feedback mechanism may exist that is yet to be elucidated. In addition, as a specific probe that detects the genetic changes of chromosome 9, p16 may not be a factor that initiates these alterations. We hypothesized that an additional potential oncogene, also located on chromosome 9, may result in cytogenetic aberration, combining the p16 with its probe and indicating a positive result. The polyploidy of chromosomes 3, 7 and 17 was also previously detected using a FISH assay $(23,24)$. It was determined that p16 amplification may exert similar biological behaviors to these genes located in chromosomes 3, 7 and 17. Secondly, the present study was retrospective and the cases that were selected may not accurately represent the population of patients with UC. In the present study, the number of patients with tumors located in the renal pelvis exceeded $50 \%$, yet renal pelvis cancer accounts for only $5 \%$ of all UC in the normal population (16). The number cases of ureter and bladder cancer is limited in the present study and the positive and negative rates of FISH results in bladder cancer were similar, which may not reflect the real condition in large sample population. At the hospital from which samples were collected in the present study, those that were recommended for FISH assays were difficult to diagnose using routine methods. The majority of patients with ureteral or bladder cancer may have been diagnosed by biopsy under ureteroscopy or cystoscopy, and therefore would not have undergone FISH-based assays for auxiliary diagnosis. Consequently, the number of cases of ureter or bladder cancer available for analysis in the current study was limited. Thus, p16 amplification appears to be significant in UC, particularly in renal pelvis cancer, although a further prospective study must be conducted for the verification of the differences observed in the present study.

Previous studies and clinical practices have combined use of chromosomes 3, 7 and 17 with the p16 (9p21) locus to detect UC by FISH assays to improve detection sensitivity $(6,8,23)$. However, in the present study, the diagnostic sensitivity of UC by FISH using a single p16 probe was higher compared with those studies using a combination of different probes. Furthermore, the present study demonstrated that p16 amplification was detected in $\sim 35 \%$ patients with UC, which may represent a key event in UC occurrence and development, and provide a novel avenue for future study. Despite the existence of studies from multiple different hospitals, no consensus has been reached on the value of FISH assays in UC diagnosis $(8,13,20,25)$. Therefore, a multi-center study with a large sample size is required. Additionally, more cases detecting other loci may also be required for future study.

In conclusion, the results of the present study indicate that p16 aberrations, including amplification, may be a primary event in the development of UC, particularly in renal pelvis cancer. As a technique for detecting cytogenetic changes of chromosomes or genes, FISH using p16 as a specific probe serves an important role in the auxiliary diagnosis and progression of patients with UC. However, increases to the sample size and follow-up duration are required in further studies.

\section{Acknowledgements}

Not applicable.

\section{Funding}

The present study was supported by grants from the National Natural Science Foundation of China (grant nos. 30900650, 81372501, 81572260, 81773299 and 31430030), the Guangdong Natural Science Foundation (grant nos. 2011B031800025, S2012010008378, S2012010008270, S2013010015327, 2013B021800126, 20090171120070, 9451008901002146, 2013B021800126, 2014A030313052, 2014J4100132, 2015A020214010, 2016A020215055 and 2013B021800259), and the Guangzhou Science and Technology Planning Program (grant no. 201704020094). 


\section{Availability of data and materials}

The analyzed data sets generated during the study are available from the corresponding author, on reasonable request.

\section{Author contributions}

ZK, LW and XM conceived and designed the study. BL, YL, SL, JZ, QH, HJ, YCh, YS, YCU, WJ and HW performed the experiments. XM and BL wrote the paper. ZK reviewed and edited the manuscript. The final version of the manuscript has been read and approved by all authors.

\section{Ethics approval and consent to participate}

The present study was approved by the Medical Ethics Committee of Sun Yat-sen University (Guangzhou, Guangdong, China). Written informed consent was obtained from all patients prior to enrollment.

\section{Consent for publication}

Not applicable.

\section{Competing interests}

The authors declare that they have no competing interests.

\section{References}

1. Chen W, Zheng R, Baade PD, Zhang S, Zeng H, Bray F, Jemal A, Yu XQ and He J: Cancer statistics in China, 2015. CA Cancer J Clin 66: 115-132, 2016.

2. Siegel RL, Miller KD and Jemal A: Cancer statistics, 2016. CA Cancer J Clin 66: 7-30, 2016.

3. Yaxley JP: Urinary tract cancers: An overview for general practice. J Family Med Prim Care 5: 533-538, 2016.

4. Tyler A: Urothelial cancers: Ureter, renal pelvis, and bladder. Semin Oncol Nurs 28: 154-162, 2012.

5. Milojevic B, Dzamic Z, Kajmakovic B, Milenkovic Petronic D and Sipetic Grujicic S: Urothelial carcinoma: Recurrence and risk factors. J BUON 20: 391-398, 2015.

6. Ho CC, Tan WP, Pathmanathan R, Tan WK and Tan HM: Fluorescence-in-situ-hybridization in the surveillance of urothelial cancers: Can use of cystoscopy or ureteroscopy be deferred? Asian Pac J Cancer Prev 14: 4057-4059, 2013.

7. Mian C, Lodde M, Comploj E, Negri G, Egarter-Vigl E, Lusuardi L, Palermo S, Marberger M and Pycha A: Liquid-based cytology as a tool for the performance of uCyt+ and Urovysion Multicolour-FISH in the detection of urothelial carcinoma. Cytopathology 14: 338-342, 2003.

8. Lin T, Liu Z, Liu L, Yang L, Han P, Zhang P and Wei Q: Prospective evaluation of fluorescence in situ hybridization for diagnosing urothelial carcinoma. Oncol Lett 13: 3928-3934 2017.
9. Reynolds JP, Voss JS, Kipp BR, Karnes RJ, Nassar A, Clayton AC, Henry MR, Sebo TJ, Zhang J and Halling KC: Comparison of urine cytology and fluorescence in situ hybridization in upper urothelial tract samples. Cancer Cytopathol 122: 459-467, 2014.

10. Nobori T, Miura K, Wu DJ, Lois A, Takabayashi K and Carson DA: Deletions of the cyclin-dependent kinase-4 inhibitor gene in multiple human cancers. Nature 368: 753-756, 1994.

11. Qin SL, Chen XJ, Xu X, Shou JZ, Bi XG, Ji L, Han YL, Cai Y, Wei F, Ma JH, et al: Detection of chromosomal alterations in bladder transitional cell carcinomas from Northern China by comparative genomic hybridization. Cancer Lett 238: 230-239, 2006.

12. Gallucci M, Guadagni F, Marzano R, Leonardo C, Merola R, Sentinelli S, Ruggeri EM, Cantiani R, Sperduti I, Lopez Fde L and Cianciulli AM: Status of the p53, p16, RB1, and HER-2 genes and chromosomes 3, 7, 9, and 17 in advanced bladder cancer: Correlation with adjacent mucosa and pathological parameters. J Clin Pathol 58: 367-371, 2005.

13. Halling KC and Kipp BR: Bladder cancer detection using FISH (UroVysion assay). Adv Anat Pathol 15: 279-286, 2008.

14. Su X, Hao H, Li X, He Z, Gong K, Zhang C, Cai L, Zhang Q, Yao L, Ding Y, et al: Fluorescence in situ hybridization status of voided urine predicts invasive and high-grade upper tract urothelial carcinoma. Oncotarget 8: 26106-26111, 2017.

15. Bubendorf L: Multiprobe fluorescence in situ hybridization (UroVysion) for the detection of urothelial carcinoma-FISHing for the right catch. Acta Cytol 55: 113-119, 2011.

16. Luo B, Li W, Deng CH, Zheng FF, Sun XZ, Wang DH and Dai YP: Utility of fluorescence in situ hybridization in the diagnosis of upper urinary tract urothelial carcinoma. Cancer Genet Cytogenet 189: 93-97, 2009.

17. Kamb A, Gruis NA, Weaver-Feldhaus J, Liu Q, Harshman K, Tavtigian SV, Stockert E, Day RS III, Johnson BE and Skolnick MH: A cell cycle regulator potentially involved in genesis of many tumor types. Science 264: 436-440, 1994.

18. Garg M: Epithelial plasticity in urothelial carcinoma: Current advancements and future challenges. World J Stem Cells 8: 260-267, 2016.

19. Amin MB: Histological variants of urothelial carcinoma: Diagnostic, therapeutic and prognostic implications. Mod Pathol 22 (Suppl 2): S96-S118, 2009.

20. Berggren de Verdier PJ, Kumar R, Adolfsson J, Larsson P, Norming U, Onelöv E, Wijkström H, Steineck G and Hemminki K: Prognostic significance of homozygous deletions and multiple duplications at the CDKN2A (p16INK4a)/ ARF (p14ARF) locus in urinary bladder cancer. Scand J Urol Nephrol 40: 363-369, 2006.

21. Ng SK, Casson RJ, Burdon KP and Craig JE: Chromosome 9p21 primary open-angle glaucoma susceptibility locus: A review. Clin Exp Ophthalmol 42: 25-32, 2014.

22. Simoneau M, LaRue HL, Aboulkassim TO, Meyer F, Moore L and Fradet Y: Chromosome 9 deletions and recurrence of supercial bladder cancer: Identication of four regions of prognostic interest. Oncogene 19: 6317-6323, 2000.

23. Wang J, Wu J, Peng L, Tu P, Li W, Liu L, Cheng W, Wang X, Zhou S, Shi S, et al: Distinguishing urothelial carcinoma in the upper urinary tract from benign diseases with hematuria using FISH. Acta Cytol 56: 533-538, 2012.

24. Sun JJ, Wu Y, Lu YM, Zhang HZ, Wang T, Yang XQ, Sun MH and Wang CF: Immunohistochemistry and fluorescence in situ hybridization can inform the differential diagnosis of low-grade noninvasive urothelial carcinoma with an inverted growth pattern and inverted urothelial papilloma. PLoS One 10: e0133530, 2015.

25. Schwarz S, Rechenmacher M, Filbeck T, Knuechel R, Blaszyk H, Hartmann A and Brockhoff G: Value of multicolour fluorescence in situ hybridisation (UroVysion) in the differential diagnosis of flat urothelial lesions. J Clin Pathol 61: 272-277, 2008. 